\title{
Working Group on Abundances in Red Giants
}

\author{
CHAIRPERSON: John C. Lattanzio \\ ORGANIZING COMMITTEE: Pavel Denissenkov, Roberto Gallino, \\ Josef Hron, Uffe Graae Jørgensen, Claudine Kahane, Sun Kwok, \\ Verne Smith, Christopher Tout, Robert F. Wing, and Ernst Zinner
}

\begin{abstract}
Determining and understanding the abundances seen in red-giant stars has taken a central role in our understanding of many branches of modern astrophysics. Activity in the area continues apace, both in terms of the fundamental physics of the stellar nucleosynthesis as well as its implications for wider fields. A major role of the Working Group has been to facilitate meetings where the fundamental role of these stars can be further understood and exploited by other researchers.
\end{abstract}

\section{Introduction and History}

The Working Group on Abundances in Red-Giants was proposed in March 2001 and formally accepted as a Working Group of Division IV in June that year. Our prime aims at the time were a) to enhance communication among interested researchers by email lists, b) to set up a new web site for posting relevant announcements, data sets, etc. and c) to prepare a proposal for a symposium during the General Assembly in Sydney. Each of these was achieved. The proposed Symposium for the GA in Sydney was not supported, but we have since then been involved in many activities including two meetings as detailed below.

\section{Organization of the Working Group}

The Organizing Committee consists of eleven scientists from nine different countries, giving us true international coverage. We also cover those discipline areas most related to the fundamental physics of red-giant nucleosynthesis, but are very much aware of the role they play in the broader context of modern astrophysics. Such a role is emphasized wherever possible.

We maintain an email list for rapid communication among interested persons. This list currently holds addresses for of 63 individuals, a little more than twice the number involved at the time of the last triennial report. These individuals represent twenty different countries. A website for the WG is maintained at

$$
\text { http: //www . maths.monash.edu.au/ johnl/wgarg/ }
$$

Some members had requested a WiKi web-page or something of that sort. We tried a "Dassie" page to help organize the meetings below but found that few people used the software, so we continue to use email as the best form of communication within the WG.

Our webpage provides links to some software developments. For example, Dr Robert Izzard is developing an abundance database and that is accessible from the WGARG page. This should be very valuable for all members of this WG. 


\subsection{Meetings}

The organization and promotion of international meetings was one of the main reasons for the formation of this WG and we have been active in this area. We have organized one two week workshop in 2005, as well as a Joint Discussion at the upcoming IAU in Prague in 2006. We are also co-sponsors of a meeting in Vienna in 2006.

\subsection{Lorentz Centre: "Nucleosynthesis in Binary Stars"}

Following a business meeting of the Working Group at the IAU GA in Sydney it was decided to apply for funding to hold a workshop at the Lorentz Centre, in Leiden. A sub-committee prepared a proposal to hold a workshop of two weeks duration at the Centre. Supplementary funding was secured from other sources in The Netherlands. It was thought that it was timely to discuss the combination of modern nucleosynthesis with the evolution of binary stars. It is likely that continued improvements in computer power will mean that some of the more long-standing problems in these areas will be approachable soon, and the workshop was designed to identify areas where effort should be directed.

The meeting took place in Leiden from April 4 to 15, and attracted 34 participants during its two-weeks. One of the areas that attracted much activity was a detailed comparison of various evolutionary codes currently in use. The results from this comparison are to be prepared for publication. The workshop allowed for summary talks of current knowledge as well as areas requiring more work, and the discussions were very active and animated. It is proposed that a follow-up meeting be held in the future.

\subsection{IAU GA in Prague: JD 11 on "Pre-Solar Grains as Astrophysical Tools"}

The analysis of pre-solar grains continues to be a rich source of data for stellar nucleosynthesis and galactic chemical evolution. To ensure that more researchers are aware of the insights provided by this field, we proposed a Joint Discussion to be held at the upcoming IAU GA in Prague in 2006. This has been approved and we are in the process of finalizing speakers and calling for contributions.

\subsection{Vienna Aug 2006: "Why Galaxies Care About AGB Stars"}

Partially inspired by some of the discussions within our WG, the group at the University of Vienna will hold a meeting just prior to the IAU GA in 2006. This meeting aims to build a bridge between AGB research and its application to the modeling of stellar populations and the chemical evolution of galaxies. Our WG is a co-sponsor of the meeting and provides one Organizing Committee member on the SOC for the meeting.

\section{Scientific Developments}

\subsection{Globular Clusters}

The various abundance anomalies in globular clusters still demand an explanation. Recent observations of fluorine show that this element can now be added to those showing variations from star-to-star within a cluster. With most of the abundance anomalies now shown to occur on the main sequence, the belief is that a pollution scenario is responsible although no consensus has yet arisen concerning the nature of the polluting stars. Although AGB stars are often cited, conclusive proof is still required, and model predictions depend on uncertainties in convection models, for example. 


\subsection{Pre-Solar Grains}

Pre-solar oxide grains provide growing evidence for extra deep mixing (also called cool bottom processing) in AGB stars. Both the large ${ }^{18} \mathrm{O}$ depletions found in many oxide grains and the high inferred ${ }^{26} \mathrm{Al} /{ }^{27} \mathrm{Al}$ ratios require processes beyond the standard stellar evolution and nucleosynthesis models for AGB stars. It is puzzling that pre-solar $\mathrm{SiC}$ grains from AGB stars have, on average, much smaller ${ }^{26} \mathrm{Al} /{ }^{27} \mathrm{Al}$ ratios than oxide grains and do not require any extra mixing processes. This observation runs counter to the expectation that, after sufficient third dredge-up, O-rich AGB stars that produced the oxide grains turn into carbon stars from which $\mathrm{SiC}$ grains can condense and that these grains also have high ${ }^{26} \mathrm{Al} /{ }^{27} \mathrm{Al}$ ratios. It might be that extra mixing keeps $\mathrm{O}$ rich stars from turning into carbon stars. However, cool bottom processing is not well understood on a fundamental level and much work remains to clarify this situation. Recent measurements of $\mathrm{Ru}$ isotopic ratios in pre-solar $\mathrm{SiC}$ grains from C-rich AGB stars found an s-process pattern except for systematic excesses in ${ }^{99} \mathrm{Ru}$. These excesses have been taken as evidence for the presence of ${ }^{99} \mathrm{Tc}$ in the grains at the time of their formation. It is satisfying that more than half a century after the astronomical observation of Tc in the atmosphere of S stars provided evidence for stellar nucleosynthesis we now find evidence for the initial presence of Tc in tiny specks of stardust we can study in the laboratory.

\subsection{Nuclear Physics}

The interaction between the astrophysics community and the nuclear physicists has perhaps never been stronger than it is at present, largely due to the demands of modern nucleosynthesis. The bi-annual meetings on "Nuclei in the Cosmos" bring together researchers from both fields, and stimulate new experiments, calculations and observations. This continues, with the next meeting in the series planned for CERN in July 2006.

\section{Looking Forward}

Red-giants continue to play a central role in many fields of modern astrophysical research. The strong interaction between nuclear physics (experimental and theoretical) and stellar astrophysics (both observational and theoretical) is proof of a vibrant working environment. The many international meetings each year attest to the activity, be it inquiry into the red-giants themselves or implications from them for other areas of astronomy. Interest in these objects shows no sign of diminishing yet.

John C. Lattanzio Chairperson of the Working Group 